\title{
Fauna de flebotomíneos (Diptera: Psychodidae) em área endêmica de leishmaniose visceral canina na região metropolitana de São Luís - MA, Brasil
}

\author{
Tatiane Aranha da Penha ${ }^{1}$ \\ Ana Clara Gomes dos Santos ${ }^{1}$ \\ José Manuel Macário Rebêlo ${ }^{2}$ \\ Jorge Luis Pinto Moraes ${ }^{2}$ \\ Rita de Maria Seabra Nogueira de Candanedo Guerra ${ }^{1 \%}$ \\ ${ }^{1}$ Universidade Estadual do Maranhão, Cidade Universitária Paulo IV \\ Caixa Postal 09, CEP 65055-000 São Luis - MA, Brasil \\ ${ }^{2}$ Universidade Federal do Maranhão, São Luis - MA, Brasil \\ * Autor para correspondência \\ grita62@hotmail.com
}

Submetido em 02/03/2012

Aceito para publicação em 15/01/2013

\section{Resumo}

Este estudo objetivou identificar a fauna de Phlebotominae (Diptera Psychodidae) em área com ocorrência de leishmaniose visceral do município de São Luís, MA. A captura dos flebotomíneos foi realizada no distrito do Tirirical, com o uso de armadilhas luminosas do tipo CDC no intradomicílio e peridomicílio (abrigo de animais) das 18 às 6 horas, uma vez por mês, em quatro pontos fixos de coleta, de agosto de 2005 a julho de 2006. Um total de 4.326 flebotomíneos (machos: $2.808 / 64,9 \%$ e fêmeas: 1.518/35,1\%) foi capturado. Sete espécies foram identificadas: Lutzomyia longipalpis (Lutz \& Neiva) (2.317/53,5\%), L. whitmani (Antunes \& Coutinho) (1.761/40,7\%), L. antunesi (Coutinho) (120/2,8\%), L. evandroi (Costa Lima \& Antunes) (99/2,3\%), L. sordellii (Shannon \& Del Ponte) (14/0,3\%), L. flaviscutellata (Mangabeira) (12/0,3\%) e L. richardwardi (Ready \& Fraiha) (3/0,1\%). Lutzomyia longipalpis e L. whitmani, as espécies mais prevalentes, ocorreram durante todo o ano. A presença de $L$. longipalpis explica a transmissão de casos autóctones de leishmaniose visceral canina. Destacase, ainda, a presença de L. whitmani e L. flaviscutellata, vetores de Leishmania braziliensis and L. amazonensis, agentes etiológicos de leishmaniose cutânea e/ou mucocutânea e leishmaniose cutânea difusa, respectivamente.

Palavras-chave: Leishmaniose; Maranhão; Phlebotominae

\section{Abstract}

Fauna of Phlebotominae (Diptera Psychodidae) in an endemic canine visceral leishmaniasis area in the metropolitan region of Sao Luis, Maranhao, Brazil. This study aimed to identify fauna of Phlebotominae (Diptera Psychodidae) in an area with occurrence of visceral leishmaniasis in the city of Sao Luis, Maranhao, Brazil. The captures of the phlebotomines was undertaken at the Tirirical district, using CDC light-traps both on an intradomiciliary and peridomiciliary basis (animals' shelter) from 6 p.m. to 6 a.m., once a month, in four fixed 
collecting points, from August 2005 to July 2006. A total of 4,326 phlebotomines (males: 2,808/64.9\%; females: 1,518/35.1\%) was captured. Seven species were identified: Lutzomyia longipalpis (Lutz \& Neiva) (2,317/53.5\%), L. whitmani (Antunes \& Coutinho)(1,761/40.7\%), L. antunesi (Coutinho) $(120 / 2.8 \%)$, L. evandroi (Costa Lima \& Antunes) (99/2.3\%), L. sordellii (Shannon \& Del Ponte) (14/0.3\%), L. flaviscutellata (Mangabeira) (12/0.3\%) and L. richardwardi (Ready \& Fraiha) (3/0.1\%). Lutzomyia longipalpis and L. whitmani, the most prevalent species, occurred throughout the year. The presence of L. longipalpis explains the transmission of autochthonous cases of canine visceral leishmaniasis. One also highlights the presence of $L$. whitmani and L. flaviscutellata, vectors of Leishmania braziliensis and L. amazonensis, etiological agents of cutaneous and/or mucocutaneous leishmaniasis and diffuse cutaneous leishmaniasis, respectively.

Key words: Leishmaniasis; Maranhao; Phlebotomines

\section{Introdução}

Os flebotomíneos são dípteros psicodídeos, vetores de espécies de protozoários parasitas do gênero Leishmania (Kinetoplastida, Trypanosomatidae), os agentes etiológicos de diferentes formas de leishmanioses, zoonoses de grande importância para a medicina humana e veterinária.

A leishmaniose visceral americana (LVA) é uma doença caracterizada pela infecção de fagócitos mononucleares por Leishmania chagasi Cunha e Chagas, que tem como principal vetor Lutzomyia longipalpis (Lutz e Neiva). Tanto a doença como o seu respectivo vetor distribuem-se do México à Argentina (YOUNG; DUCAN, 1994). Estudos epidemiológicos indicam associação entre L. chagasi e o vetor L. longipalpis. Atualmente, esta espécie vetora está presente em todas as regiões do Brasil, embora com percentuais de ocorrência diferenciados conforme a área de estudo, como demonstram os trabalhos de Oliveira et al. (2011), na região Norte, Amorá et al. (2010), no Nordeste, Souza et al. (2009), no Sul, Michalsky et al. (2009), no Sudeste, e Mestre et al. (2011), no Centro Oeste. Mudanças no comportamento humano e no ambiente natural do agente etiológico, vetor e reservatórios exercem fortes impactos no perfil epidemiológico da doença (MISSAWA; DIAS, 2007), tornando-a emergente em algumas áreas urbanas.

No município de São Luís, a doença vem sendo notificada na zona rural e em áreas periurbanas (SILVA et al., 1979; MENDES et al., 2002), sendo acompanhada invariavelmente pela presença de seu vetor, $L$. longipalpis (ARAÚJO et al., 2000; BARROS et al., 2000; CARVALHO et al., 2000; REBÊLO, 2001). Contudo os casos humanos frequentemente são antecedidos de casos caninos (BEVILACQUA et al., 2001) e, dentro desse contexto, foi detectada a ocorrência de um foco de transmissão ativa de leishmaniose visceral canina na periferia de São Luís, o que motivou a realização de um levantamento de flebotomíneos no sentido de detectar a riqueza, abundância e a distribuição mensal e sazonal das espécies, com especial atenção à ocorrência de $L$. longipalpis, o vetor do agente da doença.

\section{Material e Métodos}

$\mathrm{O}$ estudo foi realizado na localidade de Cajupe

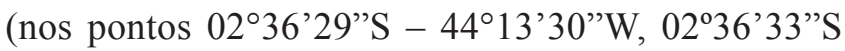

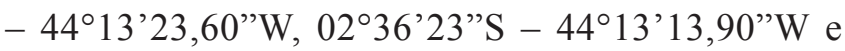
02 $36^{\prime} 20^{\prime \prime} \mathrm{S}-44^{\circ} 13^{\prime} 09^{\prime}$ 'W), no distrito do Tirirical, município de São Luís, que juntamente com os municípios de Paço do Lumiar, Raposa e São José de Ribamar situam-se na ilha de São Luís, no norte do estado do Maranhão. O povoado de Cajupe constitui uma área de transmissão ativa de leishmaniose visceral canina (de acordo com o Centro de Controle de Zoonoses) e caracteriza-se pela presença de casas construídas precariamente, com deficiência de saneamento básico, ruas sem asfalto e quintais com árvores frutíferas, que em alguns setores fazem contato com trechos de capoeiras baixas. O clima na área é o tropical mesotérmico e úmido, com duas estações bem definidas, uma chuvosa (janeiro a junho) e outra de estiagem (julho a dezembro). O índice pluviométrico é alto $(2000 \mathrm{~mm})$ e as temperaturas são elevadas durante o ano todo (média de $26^{\circ} \mathrm{C}$ ) com pequena variação anual. Os dados de pluviosidade foram obtidos no Laboratório de Geoprocessamento da Universidade Estadual do Maranhão. 
Os flebotomíneos foram coletados das 18 às 6 horas, uma vez por mês, de agosto/2005 a julho/2006, em quatro residências. Em cada uma foram instaladas duas armadilhas, uma no peridomicílio e outra dentro de casa (cozinha). O ambiente peridomiciliar foi representado por galinheiro (em três residências) e curral (uma residência). Considerando que cada armadilha funcionou durante $12 \mathrm{~h}$ consecutivas, uma vez por mês, o esforço de captura foi de $144 \mathrm{~h}$ por ano ou $1152 \mathrm{~h}$ considerando as oito armadilhas juntas.

Os espécimes coletados foram mortos por refrigeração, examinados em estereomicroscópio, separados dos demais insetos e acondicionados em frascos separados por armadilha, mês e ambiente de coleta e identificados conforme Young e Duncan (1994).

A análise estatística incluiu o teste Qui-quadrado, para verificar as diferenças entre as proporções de machos e fêmeas, ambientes de coleta e estações do ano. As diferenças foram consideradas significativas quando a probabilidade do erro foi inferior a $5 \%$ $(\mathrm{p}<0,05)$. Para a análise da abundância relativa (\%) e seus limites de confiança foi utilizado o método de Kato et al. (1952, apud LAROCA, 1995). As espécies foram consideradas dominantes quando seu limite de confiança inferior foi maior que o limite superior para espécies ausentes. Calculou-se também o índice de constância dos flebotomíneos pela fórmula: $\mathrm{C}=\mathrm{P} \times 100 / \mathrm{N}$; onde, $\mathrm{P}=$ número de coletas com a espécie estudada e $\mathrm{N}=$ número total de coletas efetuadas. Assim, as espécies foram classificadas em constantes, acessórias ou acidentais
(SILVEIRA NETO et al., 1976).

\section{Resultados}

\section{Riqueza e abundância das espécies}

Foram encontradas sete espécies de flebotomíneos: Lutzomyia antunesi, L. evandroi, L. flaviscutellata Mangabeira, L. longipalpis, L. richardwardi, L. sordellii Shannon e L. whitmani (Tabela 1).

No total foram capturados 4326 espécimes, entre machos (2808 espécimes; 64,9\%) e fêmeas (1518; $35,1 \%)$. A espécie mais abundante foi $L$. longipalpis $(53,5 \%)$, seguida por L. whitmani $(40,7 \%)$, L. antunesi (2,8\%) e L. evandroi (2,3\%). As demais espécies juntas contribuíram com $0,7 \%$.

Associação das espécies com os ambientes: A frequência dos flebotomíneos foi maior no peridomicílio (94,5\%) em comparação com o intradomicílio (5,5\%) (Tabela 1). Os machos predominaram sobre as fêmeas tanto no peridomicílio $(65,1 \%)$ como no interior das casas $(62,4 \%)$, não havendo diferença significativa entre o sexo dos flebotomíneos e o ambiente de coleta, com exceção de L. antunesi e L. sordellii, cujas fêmeas superaram os machos em ambos os ambientes e $L$. flaviscutellata que foi representada apenas por fêmeas e só foi encontrada no peridomicílio. A ordem de dominância das quatro espécies mais abundantes foi a mesma nos dois ambientes.

TABELA 1: Números de espécimes de flebotomíneos capturados no povoado de Cajupe, município de São Luís, Maranhão, Brasil, no período de agosto de 2005 a julho de 2006.

\begin{tabular}{lcccccccccc}
\hline \multicolumn{1}{c}{ Ambientes } & \multicolumn{3}{c}{ Peridomicílio } & \multicolumn{3}{c}{ Intradomicílio } & \multicolumn{3}{c}{ Total } \\
\hline \multicolumn{1}{c}{ Espécies/Sexo } & M & F & Total & M & F & Total & M & F & M+F & \% \\
\hline L. longipalpis & 1599 & 600 & 2199 & 85 & 33 & 118 & 1684 & 633 & 2317 & 53,5 \\
L. whitmani & 958 & 723 & 1681 & 46 & 34 & 80 & 1004 & 757 & 1761 & 40,7 \\
L. antunesi & 37 & 56 & 93 & 11 & 16 & 27 & 48 & 72 & 120 & 2,8 \\
L. evandroi & 63 & 28 & 91 & 5 & 3 & 8 & 68 & 31 & 99 & 2,3 \\
L. flaviscutellata & 0 & 12 & 12 & 0 & 0 & 0 & 0 & 12 & 12 & 0,3 \\
L. sordellii & 2 & 10 & 12 & 0 & 2 & 2 & 2 & 12 & 14 & 0,3 \\
L. richardwardi & 1 & 0 & 1 & 1 & 1 & 2 & 2 & 1 & 3 \\
\hline Total & 2660 & 1429 & 4089 & 148 & 89 & 237 & 2808 & 1518 & 4326 & 100,0 \\
\hline \% & 65,1 & 34,9 & 94,5 & 62,4 & 37,6 & 5,5 & 64,9 & 35,1 & 100,0 \\
\hline
\end{tabular}


Frequência mensal e estacional: Analisando a Tabela 2 observa-se que quatro espécies foram encontradas em mais de 50\% das coletas, sendo consideradas constantes, foram elas: L. longipalpis e L. whitmani presentes em onze meses $(C=91,7)$, L. evandroi presente em nove meses $(\mathrm{C}=75)$ e L. flaviscutellata em oito $(\mathrm{C}=66,7)$. No tocante a $L$. antunesi, por ter ocorrido durante seis meses $(\mathrm{C}=50)$ foi considerada acessória, e $L$. richardwardi e $L$. sordellii presentes em apenas dois meses ou em menos de $25 \%$ das coletas foram consideradas acidentais.

Analisando a distribuição das espécies por estação verifica-se que todas as sete espécies estavam presentes no período chuvoso, enquanto na estiagem não foi registrada a presença de $L$. sordellii. A maior concentração de espécies foi observada nos dois primeiros meses da estação chuvosa, janeiro e fevereiro (seis espécies), nessa mesma estação, no mês de junho, não foi possível capturar nenhum exemplar devido a chuva intensa e persistente no dia de coleta.

A abundância de indivíduos foi maior na estação chuvosa $(51,2 \%)$ do que na estiagem $(48,8 \%)$, mas essa diferença não foi estatisticamente significativa, e os meses que foram obtidas as maiores quantidades de indivíduos foram março (21\%), na estação chuvosa e agosto $(14 \%)$, na estiagem.

\section{Discussão}

A riqueza de espécies determinada neste estudo foi baixa quando comparada com aquela observada em outras localidades rurais da ilha de São Luís, onde foram encontradas uma média de dez espécies (ARAÚJO et al., 2000; BARROS et al., 2000; CARVALHO et al., 2000), contudo, na ilha inteira já foram encontradas cerca de trinta espécies, distribuídas em áreas rurais e silvestres (REBÊLO et al., 1999; MARINHO et al., 2008).

Nesse estudo, L. longipalpis foi a espécie dominante, o que explica a ocorrência de casos de leishmaniose visceral canina, e corrobora os demais estudos realizados na zona rural da ilha de São Luís, onde esse vetor tem sido capturado numa proporção de mais de sessenta por cento do total, a exemplo de outras áreas do Brasil com transmissão de LVA (BARATA et al., 2004; MONTEIRO et al., 2005; SILVA et al., 2008; SILVA; VASCONCELOS, 2005; GALATI et al., 2006; RIBEIRO; MISSAWA, 2002; SOUZA et al., 2002; TORRES et al., 2002; NERY et al., 2002; TANIGUCHI et al., 2002).

Ficou demonstrado neste estudo o predomínio de L. longipalpis no peridomicílio, o que se justifica pela presença abundante de animais domésticos, especialmente aves que funcionam como fontes sanguíneas, conforme foi observado neste estudo e em outros levados a efeito na ilha de São Luís (DIAS et al., 2003) e em outros estados (CARVALHO et al., 2010).

No tocante às demais espécies, L. whitmani e L. evandroi também têm aparecido com relativa frequência no ambiente antrópico, sobretudo no peridomicílio, sendo que a primeira merece destaque por ser incriminada no Maranhão como vetor de L. shawi

TABELA 2: Números de espécimes de flebotomíneos capturados no povoado de Cajupe, município de São Luís, Maranhão, Brasil, no período de agosto de 2005 a julho de 2006.

\begin{tabular}{|c|c|c|c|c|c|c|c|c|c|c|c|c|c|c|}
\hline \multirow{2}{*}{$\begin{array}{c}\text { Estações } \\
\text { Espécies/meses }\end{array}$} & \multicolumn{6}{|c|}{ Chuvosa } & \multicolumn{6}{|c|}{ Estiagem } & \multicolumn{2}{|c|}{ Total } \\
\hline & $\mathbf{J}$ & $\mathbf{F}$ & $\mathbf{M}$ & $\mathbf{A}$ & $\mathbf{M}$ & $\mathbf{J}$ & $\mathbf{J}$ & $\mathbf{A}$ & $\mathbf{S}$ & O & $\mathbf{N}$ & D & n & $\%$ \\
\hline L. longipalpis & 354 & 393 & 552 & 24 & 49 & - & 91 & 137 & 346 & 118 & 179 & 74 & 2317 & \\
\hline L. whitmani & 150 & 80 & 295 & 75 & 23 & - & 478 & 466 & 134 & 11 & 37 & 12 & 1761 & \\
\hline L. antunesi & 20 & 28 & 58 & 2 & - & - & 11 & - & - & - & - & 1 & 120 & \\
\hline L. evandroi & 3 & 84 & 2 & 2 & - & - & 1 & - & 1 & 2 & 2 & 2 & 99 & \\
\hline L. flaviscutellata & - & 3 & 2 & 1 & 1 & - & 1 & 2 & 1 & 1 & - & - & 12 & \\
\hline L. sordellii & 4 & 10 & - & - & - & - & - & - & - & - & - & - & 14 & \\
\hline L. richardwardi & 2 & - & - & - & - & - & - & - & - & - & - & 1 & 3 & \\
\hline Total & 533 & 598 & 909 & 104 & 73 & - & 582 & 605 & 482 & 132 & 218 & 90 & 4326 & 100,0 \\
\hline$\%$ & & & & 2 & & & & & & 48,8 & & & & 100,0 \\
\hline Pluviosidade (mm) & 205 & 216 & 272,3 & 529,8 & 299,3 & 131,8 & 45,8 & 5,5 & 1 & 3,3 & 1,3 & 130,3 & & \\
\hline
\end{tabular}


e L. braziliensis (OLIVEIRA-PEREIRA et al., 2006). Ambos parasitos já foram isolados de pacientes do oeste maranhense (FIGUEREDO et al., 1997). Quanto a $L$. antunesi e L. flaviscutellata, embora tenham sido pouco frequentes neste estudo, são abundantes no ambiente silvestre (MARINHO et al., 2008) e em capoeira baixas alteradas (BARROS et al., 2000). A última espécie merece toda a atenção, pois se trata do principal vetor da $L$. amazonensis, o agente etiológico da leishmaniose cutânea difusa; cerca de dois terços dos casos brasileiros dessa doença foram notificados no território maranhense (SALDANHA et al., 1992).

A maior proporção de machos de flebotomíneos encontrada neste estudo corrobora os resultados obtidos na maioria dos estudos envolvendo coletas de flebotomíneos com uso de armadilhas luminosas tipo CDC. Os machos naturalmente procuram as fêmeas para assegurar a cópula durante seu deslocamento (BARATA et al., 2004), não sugam sangue e são também atraídos pela fonte luminosa da armadilha. Parte das fêmeas estaria em busca de fonte alimentar e muitas, já ingurgitadas, tendem a permanecer próximas ao local de repasto devido ao peso após a alimentação e, assim, escapam da atração das armadilhas. Essa é uma hipótese razoável que explica, em parte, porque as capturas de machos são mais bem sucedidas. Obviamente, em alguns casos as fêmeas podem constituir o gênero dominante numa determinada amostragem, como ocorreu com L. flaviscutellata neste estudo, que foi representada principalmente por indivíduos que ainda não haviam sugado sangue.

Ximenes et al. (1999) observaram diferenças na proporção de machos e fêmeas para as espécies $L$. longipalpis e L. evandroi, de acordo com o animal utilizado como isca para captura manual de flebotomíneos e o uso de armadilhas luminosas em abrigo de animais. Os autores ressaltam ainda as diferenças entre a proporção de machos e fêmeas e a fonte alimentar, se animal doméstico ou silvestre.

A maioria dos estudos realizados no Maranhão enfocando a sazonalidade de flebotomíneos tem determinado a presença de várias espécies o ano inteiro, mas a abundância de indivíduos geralmente é maior no período chuvoso, que de um modo geral propicia a proliferação de insetos, devido à maior umidade, alta temperatura e disponibilidade de alimento. Este estudo não foi exceção a este padrão, embora resultados adversos possam eventualmente ocorrer motivado por um ou outro fator (REBÊLO et al., 2000). Essas características ambientais explicam também o domínio das espécies constantes e acessórias sobre as acidentais.

Em relação a $L$. whitmani, estudos desenvolvidos em áreas de cerrado (REBÊLO et al., 1999; SILVA et al., 2010) e região amazônica (REBÊLO et al., 2001) também observaram a sua ocorrência na estação seca e chuvosa, contudo com percentual maior de espécimes capturados na estação chuvosa, diferindo, portanto, da presente observação.

Em síntese, constatou-se a presença frequente e dominante de L. longipalpis na maior parte do ano em todas as residências onde os flebotomíneos foram estudados, o que explica a transmissão de parasitos causadores de leishmaniose visceral canina na área estudada. Esses dados devem ser levados em consideração na implantação de estratégias de controle vetorial, pois frequentemente, na evolução espacial de epidemia de leishmaniose visceral os casos caninos precedem os humanos. Por outro lado, a presença de $L$. flaviscutellata e $L$. whitmani não deve ser negligenciada, pois são vetores de agentes causadores de leishmaniose tegumentar.

\section{Agradecimentos}

Ao Conselho Nacional de Desenvolvimento Científico e Tecnológico/CNPq pela concessão da bolsa de iniciação científica. À Fundação Nacional de Saúde pelos equipamentos de coleta.

\section{Referências}

AMORÁ, S. A. A.; BElilAQUA, C. M. L.; FEIJÓ, F. M. C.; OliVeIRA, P. G. M.; PEIXOTO, G. C. X. ; SOUSA, R. N.; ALVES, N. D.; OLIVEIRA, L. M. B.; MACEDO, I. T. F. Sandflies (Diptera: Phlrbotominae) survey in an urban area of visceral leishmaniasis, northeastern Brazil. Revista Brasileira de Parasitologia Veterinária, São Carlos, v. 19, p. 233-237, 2010.

ARAÚJO, J. C.; REBÊLO, J. M. M; CARVALHO, M. L.; BARROS, V. L. Composição de flebotomíneos (Diptera, Psychodidae) do 
município da Raposa-MA, Brasil. Área endêmica de leishmanioses. Entomología y Vectores, Rio de Janeiro, v. 7, p. 33-47, 2000.

BARATA, R. A.; FRANÇA-SILVA, J. C.; COSTA, R. T.; FORTESDIAS, C. L.; SILVA, J. C.; PAULA, E. V.; PRATA, A.; MONTEIRO, E. M.; DIAS, E. S. Phlebotomine sand flies in Porteirinha, an area of American visceral leishmaniasis transmission in the State of Minas Gerais, Brazil. Memórias do Instituto Oswaldo Cruz, Rio de Janeiro, v. 99, p. 481-487, 2004.

BARROS, V. L.; REBÊLO, J. M. M.; SILVA, F. S. Flebotomíneos (Diptera, Psychodidae) de capoeira do município de Paço do Lumiar, estado do Maranhão, Brasil. Área endêmica de leishmanioses. Cadernos de Saúde Pública, Rio de Janeiro, v. 16, p. 265-270, 2000 .

BEVILACQUA, P. D.; PAIXÃO H. H.; MODENA, C. M.; CASTRO, M. C. P. S. Urbanização da leishmaniose visceral em Belo Horizonte. Arquivo Brasileiro de Medicina Veterinária e Zootecnia, Belo Horizonte, v. 53, p. 1-8, 2001.

CARVAlHO, G. M. L.; GONTIJO, C. M. F.; FALCÃO, A. L.; ANDRADE FILHO, J. D. Study of phlebotomine sand flies (Diptera: Psychodidae) collected in a Leishmania-endemic area of the metropolitan region of Belo Horizonte, Brazil. Journal of Medical Entomology, Lanham, v. 47, p. 972-976, 2010.

CARVAlHO, M. L.; REBÊLO, J. M. M.; ARAÚJO, J. A. C.; BARROS, V. L. Aspectos epidemiológicos dos flebotomíneos (Diptera, Psychodidae) do município de São José de Ribamar, ilha de São Luís-MA, Brasil. Área endêmica de leishmanioses. Entomologia y Vectores, Rio de Janeiro, v. 7, p. 19-32, 2000.

DIAS, F. O. P.; LOROSA, E. S.; REBELO, J. M. M. Fonte alimentar sangüínea e a peridomiciliação de Lutzomyia longipalpis (Lutz \& Neiva, 1912) (Psychodidae, Phlebotominae). Cadernos de Saúde Pública, Rio de Janeiro, v. 19, p. 1373-1380, 2003.

FIGUEREDO, F. V.; CUNHA A. K.; GAMA M. E. A.; COSTA, J. M. L.. Leishmaniose Tegumentar Americana (LTA) em área endêmica do estado do Maranhão. In: CONGRESSO DA SOCIEDADE BRASILEIRA DE MEDICINA TROPICAL, 33, 1997, Belo Horizonte. Resumos... Belo Horizonte: Sociedade Brasileira de Medicina Tropical, 1997. Versão eletrônica.

GALATI, E. A. B.; NUNES, V. L. B.; BOGGIANI, P. C.; DORVAL, M. E.; CRISTALDO, G.; ROCHA, H. C.; OSHIRO, E. T.; DAMASCENO-JÚNIOR, G. A. Phlebotomines (Diptera: Psychodidae) in forested areas of the Serra da Bodoquena, state of Mato Grosso do Sul, Brazil. Memórias do Instituto Oswaldo Cruz, Rio de Janeiro, v. 101, p. 175-193, 2006.

KREBS, C. J. Ecological methodology. New York: Harper \& Hall, 1989. $654 \mathrm{p}$.

LAROCA, S. Ecologia: Princípios e métodos. Petrópolis: Vozes, 1995. $197 \mathrm{p}$.

MARINHO, R. M.; FONTELES, R. S.; VASCONCELOS, G. C.; AZEVÊDO, P. C. B.; MORAES, J. L. P.; REBÊLO, J. M. M. Flebotomíneos (Diptera, Psychodidae) em reservas florestais da área metropolitana de São Luis, Maranhão, Brazil. Revista Brasileira de Entomologia, Curitiba, v. 52, p. 112-116, 2008.

MENDES, W. S.; SILVA, A. A. M.; TROVÃO, J. R.; SILVA, A. R.; COSTA, J. M. L. Expansão espacial da leishmaniose visceral americana em São Luis, Maranhão, Brasil. Revista da Sociedade Brasileira de Medicina Tropical, Uberaba, v. 35, p. 227-231, 2002.
MESTRE, G. L. C.; RIBEIRO, A. L. M.; MIYAZAKI, R. D.; RODRIGUES, J. S. V.; ALMEIDA, A. B. F.; SOUSA, V. R. F.; MISSAWA, N. A. Phlebotominae sand flies and canine infection in áreas of human visceral leishmaniasis, Cuiabá, Mato Grosso. Revista Brasileira de Parasitologia Veterinária, São Carlos, v. 20, p. 228-234, 2011.

MICHASKY, E. M.; FRANÇA-SILVA, J. C.; BARATA, R. A.; SILVA, F. O. L.; LOUREIRO, A. M. F.; FORTES-DIAS, C. L.; DIAS, E. S. Phlebotominae distribution in Janaúba, na área of transmission for visceral leishmaniasis in Brazil. Memórias do Instituto Oswaldo Cruz, Rio de Janeiro, v. 104, p. 56-61, 2009.

MISSAWA, N. A.; DIAS, E. S. Phlebotomine sand flies (Diptera: Psychodidae) in the municipality of Várzea Grande: an area of transmission of visceral leishmaniasis in the state of Mato Grosso, Brazil. Memórias do Instituto Oswaldo Cruz, Rio de Janeiro, v. 102, p. 913-918, 2007.

MONTEIRO, E. M.; FRANÇA-SILVA, J. C.; COSTA, R. T.; COSTA, D. C.; BARATA, R. A.; PAULA, E. V.; MACHADOCOELHO, G. L. L.; ROCHA, M. F.; FORTES-DIAS, C. L.; DIAS, E. S. Leishmaniose visceral: estudo de flebotomíneos e infecção canina em Montes Claros, Minas Gerais. Revista da Sociedade Brasileira de Medicina Tropical, Uberaba, v. 38, p. 147-152, 2005.

NERY, L. C. R.; FREITAS, R. A.; FRANCO, A. M. R. Diversity of phebotominae sandflies (Diptera: Psychodidae) in the campus of the Amazonas university, Manaus, AM. Entomologia y Vectores, Rio de Janeiro, v. 9, p. 117, 2002.

OLIVERIA-PEREIRA, Y. N.; REBÊLO, J. M. M.; MORAES, J. L. P.; PEREIRA, S. R. F. Diagnóstico molecular da taxa de infecção natural de flebotomíneos (Psychodidae, Lutzomyia) por Leishmania sp. na Amazônia maranhense. Revista da Sociedade Brasileira de Medicina Tropical, Uberaba, v. 39, p. 540-543, 2006.

OLIVEIRA, D. M. S.; SARAIVA, E. M.; ISHIKAWA, E. A. Y.; SOUSA, A. A. A.; SILVA, E. O.; SILVA, I. M. Distribution of phlebotomine fauna (Diptera: Psychodidae) across na urban - rural gradient in na área of endemic visceral leishmaniasis in northern Brazil. Memórias do Instituto Oswaldo Cruz, Rio de Janeiro, v. 106, p. 1039-1044, 2011.

PIELOU, E. C. Biological diversity. New York: John Wiley \& Sons, 1975. 165 p.

REBÊLO, J. M. M. Frequência horária e sazonalidade de Lutzomyia longipalpis (Diptera: Psychodidae: Phlebotominae) na ilha de São Luis, Maranhão, Brasil. Cadernos de Saúde Pública, Rio de Janeiro, v. 17, p. 221-227, 2001.

REBÊLO, J. M. M.; ARAÚJO, J. C.; CARVALHO, M. L.; BARROS, V. L. L.; SILVA, F. S.; OLIVEIRA, S. T. Flebótomos (Diptera, Phlebotominae) da ilha de São Luís, zona do Golfão Maranhense, Brasil. Revista da Sociedade Brasileira de Medicina Tropical, Uberaba, v. 32, p. 247-253, 1999.

REBÊLO, J. M. M.; OLIVEIRA, S. T.; BARROS, V. L. L.; COSTA, J. M. L. sandflies (Diptera: Pshychodidae) of the Amazonia of Maranhão. V. Seasonal occurrence in ancient colonization area and endemic for cutananeous leishmaniosis. Revista Brasileira de Parasitologia, São Carlos, v. 61, p. 107-115, 2001.

REBÊLO, J. M. M.; OLIVEIRA, S. T.; BARROS, V. L. L.; SILVA, F. S.; COSTA, J. M. L.; FERREIRA, L. A.; SILVA, A. R. Phlebotominae (Diptera: Psychodidae) de Lagoas, município 
de Buriticupu, Amazônia Maranhense. I - riqueza e abundância relativa das espécies em área de colonização recente. Revista da Sociedade Brasileira de Medicina Tropical, v. 33, p. 11-19, 2000. RIBEIRO, A. L. M.; MISSAWA, N. A. Spatial distribution of phlebotominae species in the state of Mato Grosso/Brazil in the period of 1996 to 2001. Entomologia y Vectores, Rio de Janeiro, v. 9, p. 33, 2002.

SALDANHA, A. C. R.; SILVA, A. C. M.; GALVÃO, C. E. S.; SILVA, C. M. P.; COSTA, J. M. L. Procedência de pacientes portadores de leishmaniose cutânea difusa (LCD) no estado do Maranhão - Brasil. Revista da Sociedade Brasileira de Medicina Tropical, Uberaba v. 25, p. 271-273, 1992.

SILVA, A. M.; CAMARGO, N. J.; SANTOS, D. R.; MASSAFERA, R.; FERREIRA, A. C.; POSTAI, C.; CRISTÓVÃO, E. C.; KONOLSAISEN, J. F.; BISETTO JR, A.; PERINAZO, R.; TEODORO, U.; GALATI, E. A. B. Diversidade, distribuição e abundância de flebotomíneos (Diptera: Psychodidae) no Paraná. Neotropical Entomology, Piracicaba, v. 37, p. 209-225, 2008.

SILVA, F. S.; CARVALHO, L. P. C.; CARDOZO, F. P.; MORAES, J. L. P.; REBÊLO, J. M. M. Sand flies (Diptera: Psychodidae) in the cerrado área of the Maranhão state, Brazil. Neotropical Entomology, Piracicaba, v. 36, p. 1032-1038, 2010.

SILVA, A. R.; MARTINS, G.; MELO, J. E. M.; ARAÚJO, J. P; MENDES, M. G. Surto epidêmico de leishmaniose tegumentar americana na colonização agrícola de Buriticupu (MA). Revista do Instituto de Medicina Tropical de São Paulo, São Paulo, v. 21, p. 45-50, 1979.

SILVA, D. F.; VASCONCELOS, S. D. Flebotomíneo em fragmentos de mata atlântica na região metropolitana de Recife, PE. Revista da Sociedade Brasileira de Medicina Tropical, Uberaba, v. 38, p. 264-266, 2005.
SILVEIRANETO, S.; NAKANO, O.; BARBIN, D.; VILLANOVA, N. A. Manual de ecologia dos insetos. São Paulo: Agronômica Ceres, 1976. 419 p.

SOUZA, G. D.; SANTOS, E.; ANDRADE FILHO, J. D. The first report of the main vector of visceral leishmaniasis in America, Lutzomyia longipalpis (Lutz \& Neiva) (Diptera: Psychodidae: Phlebotominae), in the state of Rio Grande do Sul, Memórias do Instituto Oswaldo Cruz, Rio de Janeiro, v. 104, p. 1181-1182, 2009.

SOUZA, N. A.; COELHO, C. A. A.; VITELA, M. L.; PEIXOTO, A. A.; RANGEL, E. F. Ecology of sandfly vectors in an american cutaneous leishmaniasis (ALC) endemic area in state of Rio de Janeiro, Brazil. Entomología y Vectores, Rio de Janeiro, v. 9, p. $105,2002$.

TANIGUCHI, H. H.; TOLEZANO, J. E.; LAROSA, R.; ELIAS, C. R.; GALATI, E. A. B. Sandfly ecological observations in Eldorado county, Ribeira valley, São Paulo state, Brazil, period between 1996 and 1997. Entomología y Vectores, Rio de Janeiro, v. 9, p. 116, 2002.

TORRES, W. M. P.; FREITAS, R. A.; BARRET, T. V. Phlebotominae of the lower Araguaia region of Para, north Brazil. Entomología $y$ Vectores, Rio de Janeiro, v. 9, p. 48, 2002.

XIMENES, M. F. F. M.; SOUZA, M. F.; CASTEllón, E. G. Density of sand flies (Diptera: Psychodidae) in domestic and wild animal shelters in a area of visceral leishmaniasis in the state of rio Grande do Norte, Brazil. Memórias do Instituto Oswaldo Cruz, Rio de Janeiro, v. 94, p. 427-432, 1999.

YOUNG D. C.; DUCAN, N. A. Guide to the identification and geographic distribution of Lutzomyia sandflies in Mexico, the West Indies, Central and South America (Diptera: Psychodidae). Memories of the American Entomology Institute, Gainesville, v. 54, p. 1-881, 1994. 